\title{
New Insights in Synthetic Fiber Rope Elongation and its Detection for Ultra Lightweight Tendon Driven Series Elastic Robots
}

\author{
Jérôme Kirchhoff and Oskar von Stryk
}

\begin{abstract}
Selecting transmission components for tendon driven actuation systems can be challenging because of the variety of available solutions. Synthetic fiber ropes have great potential for these systems, but the typically provided characteristics are not always suitable to decide whether a rope should be used in the specific system or not. In this paper, a comparative evaluation of the elongation characteristic regarding different rope materials, manufacturers and temperatures is presented. Further, the influence of guiding pulleys is investigated concerning the application at ultra lightweight tendon driven series elastic robots. The knowledge gained from the performed new rope analysis supports the design process of tendon driven robots. Since tendon elongation influences the control performance and joint torque estimation of the regarded class of robots, a novel observer-based approach for tendon elongation detection is presented and evaluated, which enables to monitor wear for quality assurance and to avoid failures.
\end{abstract}

\section{INTRODUCTION}

One of the current trends in industrial automation is bringing humans and robots together to perform a task in the same workspace without fences. This can gain productivity, since the automation process can benefit from the human's dexterity and process knowledge, as well as from the robot's repetitive performance without tiredness.

Safe physical human-robot interaction (pHRI) is crucial in this scenario. The kinetic energy that can be transferred during a collision without severe injuries is bounded by the human's biomechanical limits [1]. To stay within these limits, even small joint elasticities can be introduced in the joint to decouple the motor and link inertia, causing a maximum transient collision force determined only by the link inertia, and further, reducing the robot's effective mass.

A typical approach to develop a robot that is capable for pHRI is to downscale industrial robot arms with motors still placed in the joints. This reduces the robot's effective mass but is limited by reach and payload. Introducing tendons in the robot's mechanical design enables to shift the motors away from the joints towards the base, which results in a different class of lightweight robots.

Besides industrial automation, tendons can also be relevant for other fields of human near applications. Reproducing human or animal like walking can benefit from the use of tendons. Even if their use is only passive without actuation, it is possible to realize a joint coupling more similar to the biomechanical mechanisms which helps to synchronize the joints and facilitates the control task [2]. Also for wearable

The authors are with the Simulation, Optimization and Robotics Group, Department of Computer Science, Technische Universität Darmstadt, Germany [kirchhoff, stryk] @sim.tu-darmstadt.de robots, e.g., to support elderly people performing a motion or new types of orthoses, tendons can help to create a more comfortable and lightweight structure.

Independent of the specific application, tendons can be implemented in different ways. For instance belts or steel cables can be used to move joints, as done in some industrial robots. But these solutions are not necessarily suitable for structures where pulleys with small radii are used and high forces have to be transmitted. In these cases, synthetic ropes should be considered. In the last decade the evolution in synthetic fiber materials and production processes led to products with impressing strength (see Table I).

Since the scale of robots in the vicinity of humans is limited by safety and weight, also the used pulleys and thus rope diameter is limited. Synthetic ropes with small diameters are typically used in the area of sailing and sport activities that use kites or parachutes. Here, the relevant parameters are the rope's diameter, breaking strength and weight. But these are not always suitable to decide about the utilization potential in a complex mechatronical system where a rope is wound around multiple pulleys. Especially, the irretrievable rope elongation characteristic (creep) is a relevant factor that should be known and continuously monitored because of the negative influence on the joint position control performance.

The contribution of this work consists of

- new comparative creep experiments regarding different fiber materials, manufacturers, and diameters,

- the experimental evaluation of the influence of a different amount of pulleys on commonly used HMPA fiber,

- and an observer-based approach for tendon elongation detection to monitor wear for quality assurance.

The performed experiments gives new insights into the rope elongation characteristics, that are typically not provided by the rope manufacturers.

\section{RELATED WORK AND BACKGROUND}

Using ropes as tendons in robotic systems provides the opportunity of small scale mechanical design, because of the high flexibility, high strength to weight ratio, and possible small bending radius. That is the reason why they are used for example in systems like anthropomorphic hands [3]-[5], bipedal robots [6], [7], small size ultra lightweight robot arms [8]-[10] or novel actuation approaches [11].

Synthetic ropes can be braided in different ways using different fibers. These fiber materials have evolved in the last decades improving the strength to weight ratio and reducing creep effects. A comparison of rope constructions and fiber 
This is the author's version of an article that has been published in the Proceedings of the

IEEE International Conference on Advanced Intelligent Mechatronics (AIM), Munich, July 3-7, 2017, pp. 64-69.

The final version of record is available at https://doi.org/10.1109/AIM.2017.8013996

\begin{tabular}{|c|c|c|c|c|c|c|c|}
\hline Manufacturer & Product & Fiber & Material & Diameter & Breaking Load & Plait & Weight \\
\hline Gottifredi Maffioli & SK99 Ultra & Dyneema SK99 & HMPE & $2.00 \mathrm{~mm}$ & $560 \mathrm{daN}$ & - & $2.50 \mathrm{~g} / \mathrm{m}$ \\
\hline Liros & Aramid Braided Cord & Aramid & Aramid & $2.00 \mathrm{~mm}$ & $250 \mathrm{daN}$ & 16-plaited & - \\
\hline Liros & DC000-0301 & Dyneema SK99 & HMPE & $1.50 \mathrm{~mm}$ & $445 \mathrm{daN}$ & 12-plaited & $1.45 \mathrm{~g} / \mathrm{m}$ \\
\hline Liros & DC000-0701 & Dyneema SK99 & HMPE & $2.00 \mathrm{~mm}$ & $700 \mathrm{daN}$ & 12-plaited & $3.05 \mathrm{~g} / \mathrm{m}$ \\
\hline Ockert & Climax Combat & Dyneema SK75 & HMPE & $1.22 \mathrm{~mm}$ & $210 \mathrm{daN}$ & - & - \\
\hline Teufelberger & FL-32.NG & Dyneema DM20 & HMPE & $1.50 \mathrm{~mm}$ & $350 \mathrm{daN}$ & 8-plaited & $1.80 \mathrm{~g} / \mathrm{m}$ \\
\hline Teufelberger & FL-9.NG & Dyneema SK75 & HMPE & $2.05 \mathrm{~mm}$ & $650 \mathrm{daN}$ & 12-plaited & $3.30 \mathrm{~g} / \mathrm{m}$ \\
\hline TU Chemnitz & - & Technora Black & Aramid & $1.80 \mathrm{~mm}$ & - & - & - \\
\hline TU Chemnitz & - & Vectran & LCP & $1.80 \mathrm{~mm}$ & - & - & - \\
\hline
\end{tabular}

TABLE I

LIST OF EVALUATED ROPES PRODUCED BY VARIOUS MANUFACTURERS IN A DIAMETER RANGE FROM 1.22MM TO 2.05MM, A BREAKING LOAD OF AT LEAST 210DAN PRODUCED WITH USUALLY UTILIZED FIBERS. ALL INFORMATION HAVE BEEN PROVIDED BY THE MANUFACTURERS.

characteristics can be found in [12]. For the application of tendon driven robots, ropes need to have high strength at low elongation and be resistant to abrasion. The ropes regarded in this paper meet these requirements and consists of High Modulus Polyethylene (HMPE), Liquid Crystal Polymer (LCP) and Aromatic Polyamide (Aramid). As presented in [12], the Polybenzoxazole (PBO) fiber shows the lowest elongation at break but has low abrasion resistance [13] and is therefore not regarded.

Besides general fiber characteristics, it is important to investigate the rope's application-dependent performance. To abstract from the specific scenario, different experiments can be performed, as a tensile creep test where a constant force is exerted to the rope and the elongation over time at a certain temperature is measured, a bending fatigue test where a rope is cycled over a sheave under a constant tension, or winding the rope to investigate the contact effects. The test results of such tests are typically not provided by the specific rope manufacturer.

Since the production process and braid can influence the rope's characteristics, we present a comparative evaluation of different ropes using a tensile creep test and a test similar to a bending fatigue test but winding the rope, using a different number of guiding pulleys and loads, according to the application in tendon driven robots. To the authors knowledge, such analysis has not yet been performed, but is required for the design process of tendon driven robots. Further, an observer-based approach will be presented to estimate the rope elongation during its life cycle.

\section{ROPE EXPERIMENTS}

As described in Section I, the creep characteristics of ropes are a relevant factor if they are used to move parts that have to be accurately position controlled. In order to compensate the irreversible tendon elongation,the actuator position has to be adjusted appropriately. Thus, a rope with less creep is desirable for the specific application. Since information about the creep characteristics are typically not available for the end products but only qualitative characteristics for the raw fibers [12], these have to be identified by experiment.

In this chapter the two types of performed experiments are described. The First experiment has been carried out in cooperation with the State Materials Testing Institute (MTI)
Darmstadt (area of expertise plastics and composites) comparing ropes of different manufactures, fibers and diameters at a specific operating point. As second experiment, a rope is fixed in a test setup to move a revolute joint via different types and numbers of pulleys. This experiment aims to be closer to the usage in a real mechatronic system.

\section{A. Evaluated Ropes}

The set of ropes used in the experiments results from a market research of ropes with a diameter of $1.22-2.05 \mathrm{~mm}$ and a breaking load of at least $200 \mathrm{daN}$. These values are determined by the development experience with tendon driven robots ([10], [7]) where small pulley radii are limiting the upper diameter range and also causing high rope forces when used in the drive shaft. Further, only ropes have been chosen for which at least a minimum number of parameters (fiber type, diameter, breaking load) are provided. The selected manufacturers are mainly from Germany but using customary fibers. For additional fiber comparison two ropes from the Technical University (TU) Chemnitz provided by the MTI are considered in the test set. All ropes are listed in Table I.

\section{B. Tensile Creep Experiments}

The tensile creep experiments are performed following [14] under standard atmosphere 23/50 [15]. A force of $60 \mathrm{~N}$ is applied to the rope pieces of $350 \mathrm{~mm}$ length during $85 \mathrm{~min}$. The applied force is kept constant over the whole experiment while simultaneously measuring the rope elongation. The same force has been used for all ropes to compare their elongation characteristic according to one defined operating point, since this is typically necessary if a rope for a specific application has to be chosen. This experiment has been repeated for a subset of ropes in a heat cabinet with $60^{\circ} \mathrm{C}$.

The experimental results of the tensile creep experiment under $23^{\circ} \mathrm{C}$ are depicted in Fig. 1 and discussed in this paragraph. The parameter $m_{E}$ shown in the legend represents the slope of the curve starting at $20 \mathrm{~min}$ created by fitting a linear polynomial. Only looking at the rope's diameter and breaking load, one could expect that the $2 \mathrm{~mm}$ ropes (except the Aramid fiber because of the low breaking load) will outperform the other ones. But this is not the case, as can be seen with the Gottifredi Maffioli SK99 Ultra rope, although it consists of a new generation of the Dyneema fiber SK99. 
This is the author's version of an article that has been published in the Proceedings of the

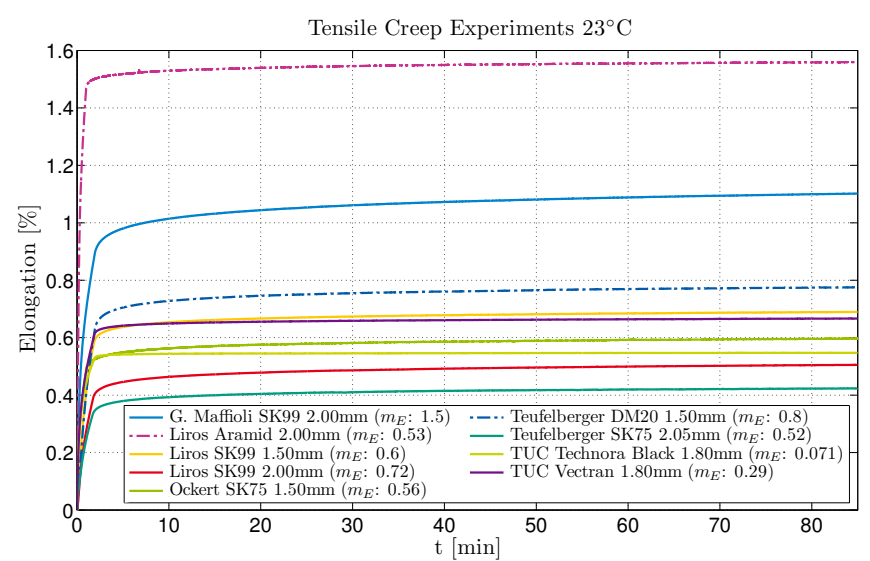

Fig. 1. Elongation results of the tensile creep experiments exerting a constant force of $60 \mathrm{~N}$ to the ropes with $350 \mathrm{~mm}$ length at $23^{\circ} \mathrm{C}$

Comparing the Aramid fiber rope with the Ockert Climax Combat that has a rather similar breaking load, shows a final elongation that is more than half as large as smaller diameters. The elongation slopes of these ropes are almost the same. The final elongation of the set of ropes with a diameter smaller that $2 \mathrm{~mm}$ is within a range of almost $0.2 \%$. Besides this, the two fibers Technora Black and Vectran provided by (MTI) show less elongation slope than the other ropes.

In the second tensile creep test setup, the environment temperature has been increased to $60^{\circ} \mathrm{C}$. The experiments were performed for the four different fibers DM20 (HMPE), SK99 (HMPE), Technora Black (Aramid) and Vectran (LCP). Since $S K 75$ and SK99 are evolutions of HMPE fibers, only one rope consisting of $S K 99$ is regarded. The results, as illustrated in Figure 2, show that all ropes except the Liros SK99 reaches an elongation similar to $23^{\circ} \mathrm{C}$. Further, the Technora Black and Vectran fiber present only a low incrementation of the elongation slope. In contrast to this, the SK99 and DM2O fiber largely decrease the elongation slope. It is noticeable that the elongation of the Liros SK99 rope finally reaches a value that is only half of the value measured at $23^{\circ} \mathrm{C}$. One possible explanation for this behavior is that the increased temperature facilitates the fiber molecules to reach a state of higher entropy, which acts against the elongation. This meets the statement in [12] that HMPE fibers show degradation at a temperature higher than $65^{\circ} \mathrm{C}$.

Summarizing the tensile creep experiments, all ropes show an asymptotic saturation in elongation with a low but not neglectable elongation slope. Further, the production process and the way how a rope is braided changes the elongation behavior more than can be represented by the commonly provided breaking force, especially if the operating point is far below. The difference between the Aramid ropes is remarkable high, whereas the Technora Black and Vectran fibers are really promising in particular because of the low elongation slope.

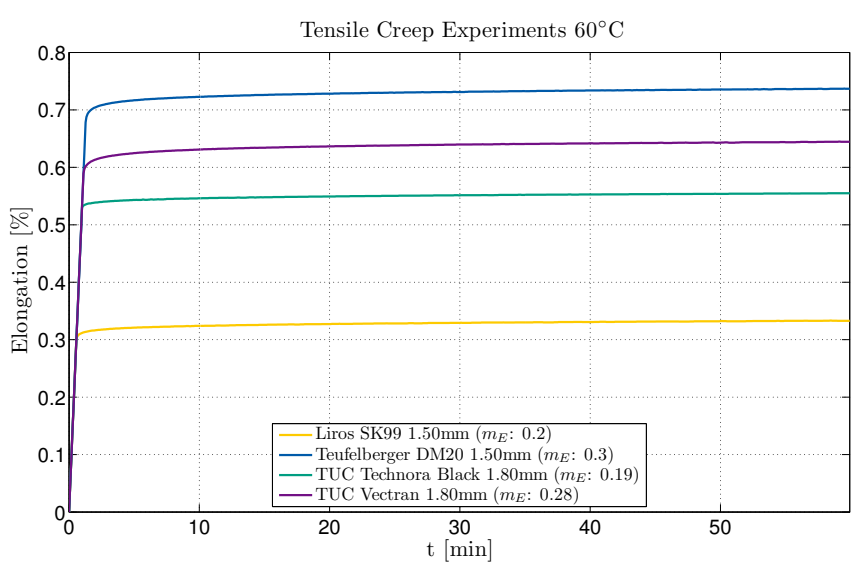

Fig. 2. Elongation results of the tensile creep experiments exerting a constant force of $60 \mathrm{~N}$ to the ropes with $350 \mathrm{~mm}$ length at $60^{\circ} \mathrm{C}$

\section{Bending Experiments}

Using ropes in the drive train of an actuated system introduces further influences regarding the rope's elongation behavior. Typically, a rope is guided by pulleys to transmit a force. These guiding pulleys exert a force on the braided rope structure that is orthogonal to the transmitted force. In order to investigate the influence of these guiding pulleys on the elongation characteristics, the test bench as shown in Fig. 3 is used.

The test bench consists of the electrical part with the power supply, power electronics, DC motor, and EtherCAT connection, as well as the mechanical part to guide the rope in different ways from the actuated drive pulley to the rotary joint pulley, see Fig. 3 (left). The motor is mounted on a moveable carriage that can be pulled with a defined force. The rope elongation is measured via the movement of the carriage using a half millimeter scale and a camera. Four different rope routings (A)-(D) are examined, see Fig. 3 (right). As reference experiment, the rope connects the drive pulley with the joint pulley without additional guiding. After this, three guiding pulleys in different arrangements from low to high rope bending (wrap angle) are introduced.

During the experiments, the motor rotates in a sinusoidal motion in a way that a length of approximately $10 \mathrm{~cm}$ of the rope is repeatedly unrolled. A force of $200 \mathrm{~N}$ continuously pulls the carriage that ideally results in a force of $100 \mathrm{~N}$ in each rope. Experiment (B) only slightly bends the rope and consists of a small wrap angle in each guiding pulley. The distance between the pulleys is larger than the unrolled rope length, thus, the stressed rope segments are distinct. In experiment $(\mathrm{C})$ one guiding pulley is placed in a way that the rope is bent more than $180^{\circ}$, with again distinct stressed rope segments. This is changed in experiment (D). Here, the three guiding pulleys are placed nearby, so that a portion of the rope moves around each pulley. The experiments are performed for four hours, two times each.

During the bending experiments the Liros SK99 with commonly available HMPE fiber is used. The determined elongation characteristics are shown in Fig 4. As expected, 

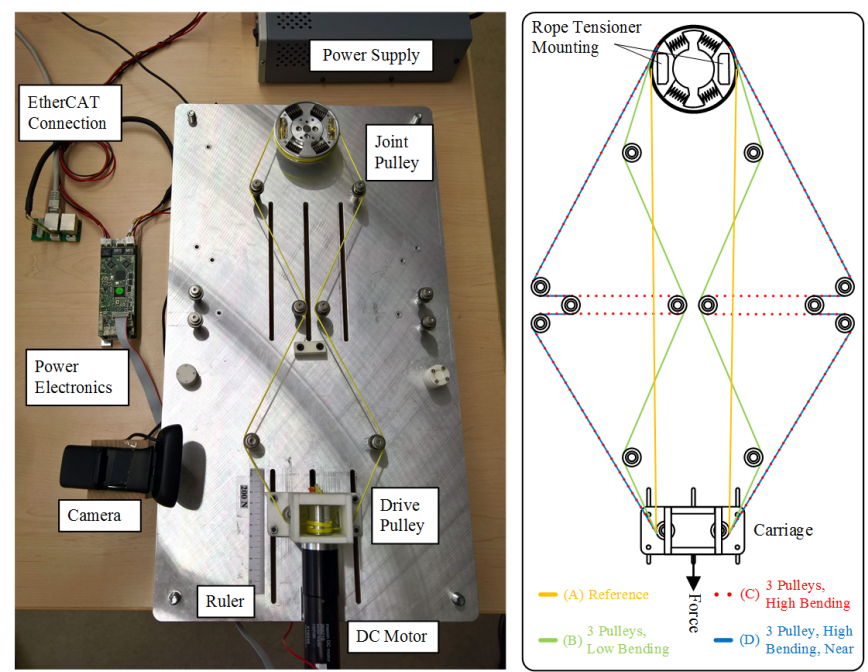

Fig. 3. Test bench for bending test execution. The movement of the carriage is recorded using the camera for four different rope routings (A)-(D), while the rope is periodically moved rotating the joint pulley, with a constant force pulling the carriage.

the reference experiment (A) causes the lowest elongation. Surprisingly, experiment (B), containing the lowest rope bending, reaches the highest elongation. Concerning the high bending experiments (C) and (D), one can see that stressing a rope portion more than once with a guiding pulley, results in a higher elongation. The high elongation of experiment (B) could be explained with the low wrap angle at each pulley that also reduces the influence of the rope friction which keeps the rope forces almost unchanged, but additionally every pulley stresses the braided structure. Analogue to the experiments without any bending in the previous chapter, the characteristics asymptotically approach an elongation value over time.

Since in a real robotic system the forces exerted on the ropes are dynamically changing, we investigated the rope elongation with changing force in a basic manner. For this purpose two experiments were performed with a force of $200 \mathrm{~N}$ followed by a reduced force of $120 \mathrm{~N}$ pulling at the carriage $(\mathrm{E})$, and vice versa $(\mathrm{F})$. The forces changed after 80min. The elongation results are shown in Fig. 5. Two characteristics for HMPE fiber ropes can be observed here. Decreasing the force in experiment (E) leads to a reduction in elongation, but preserving an irreversible elongation. Increasing the force (see experiment $(\mathrm{F})$ ) will also increase elongation but during a longer time period.

This investigation makes it reasonable to pretension the ropes according to the typical workload. In this way, one should be able to reduce the effect of elongation. Further, dynamic forces that are higher than the regular work load seems to be at least partially compensated if the force reduces again.

\section{Tendon Elongation Estimation}

In tendon driven joint elastic ultra lightweight robotic systems the rope elongation has a crucial influence on the

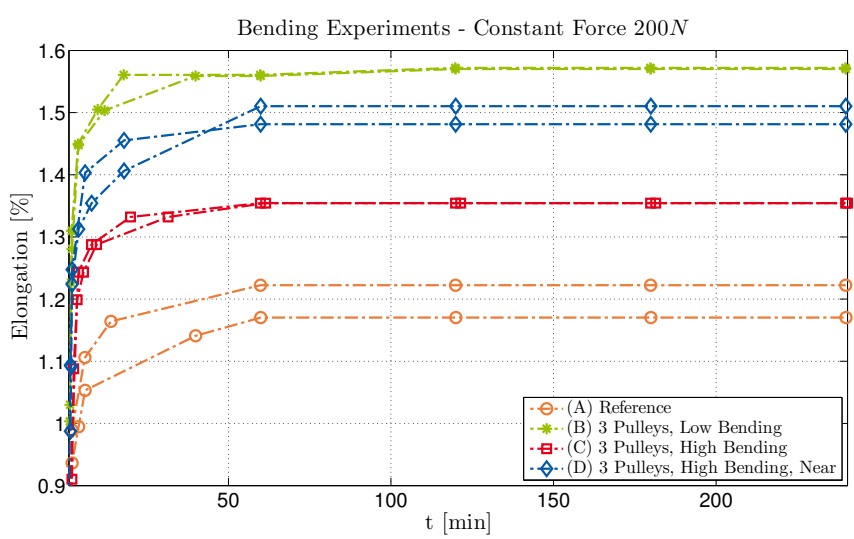

Fig. 4. Elongation results of the bending experiments exerting a constant force of $200 \mathrm{~N}$ to the ropes during $240 \mathrm{~min}$. Each experiment is performed two times (same line color).

control performance, since the elongation has to be compensated using an appropriate desired motor position to reach the targeted joint position. Further, the long term elongation information is needed to detect the need of maintenance, e.g. to replace the rope before it breaks. In this chapter a general elongation estimation scheme is presented that only needs motor and joint position sensor information.

The reduced dynamics model [16] of a joint elastic robot arm can be represented as:

$$
\begin{aligned}
\boldsymbol{M}(\boldsymbol{q}) \ddot{\boldsymbol{q}}+\boldsymbol{C}(\boldsymbol{q}, \dot{\boldsymbol{q}}) \dot{\boldsymbol{q}}+\boldsymbol{g}(\boldsymbol{q})+\boldsymbol{\tau}_{f, j} & =\boldsymbol{\tau}_{e l}+\boldsymbol{\tau}_{e x t} \\
\boldsymbol{I}_{m} \ddot{\boldsymbol{\theta}}+\boldsymbol{\tau}_{f}+\boldsymbol{\tau}_{e l} & =\boldsymbol{\tau}_{m} \\
\boldsymbol{K}(\boldsymbol{\theta}-\boldsymbol{q}) & =\boldsymbol{\tau}_{e l}
\end{aligned}
$$

with the joint positions $\boldsymbol{q} \in \mathbb{R}^{n}$, its time derivatives $\dot{\boldsymbol{q}}$ and $\ddot{\boldsymbol{q}}$, the motor position $\boldsymbol{\theta} \in \mathbb{R}^{n}$ and acceleration $\ddot{\boldsymbol{\theta}}$, the mass matrix $\boldsymbol{M} \in \mathbb{R}^{n \times n}$, the matrix $C \in \mathbb{R}^{n \times n}$ of the centrifugal and Coriolis term, the gravity torque vector $\boldsymbol{g} \in \mathbb{R}^{n}$, the elastic joint torque vector $\boldsymbol{\tau}_{e l} \in \mathbb{R}^{n}$, the external disturbance torque vector $\boldsymbol{\tau}_{e x t} \in \mathbb{R}^{n}$, the drive train friction vector $\boldsymbol{\tau}_{f} \in \mathbb{R}^{n}$, the joint friction vector $\boldsymbol{\tau}_{f, j} \in \mathbb{R}^{n}$, the motor torque vector $\boldsymbol{\tau}_{m} \in \mathbb{R}^{n}$, the diagonal motor and gear inertia matrix $\boldsymbol{I}_{m} \in \mathbb{R}^{n \times n}$, the diagonal elastic transmission stiffness matrix $\boldsymbol{K} \in \mathbb{R}^{n \times n}$, and $\mathrm{n}$ being the degree of freedom.

If the tendon elongates, a motor position offset $\boldsymbol{\Delta}_{e} \in \mathbb{R}^{n}$ is introduced changing the elastic joint torque estimation to

$$
\hat{\boldsymbol{\tau}}_{e l}=\boldsymbol{K}(\underbrace{\boldsymbol{\theta}+\boldsymbol{\Delta}_{e}}_{\hat{\boldsymbol{\theta}}}-\boldsymbol{q}),
$$

with $\hat{\boldsymbol{\theta}}$ being the measured motor position including the position offset.

As the elongation increases during the life time of the rope, the estimated joint torque increases as well (see (4)). In order to realize an accurate control performance the control approach presented in [17] can be used. Besides the control performance, also the physical interaction is negatively influenced by an incorrect joint torque estimation, since the estimation of external torques based on the dynamics model ([18], [19]) deviates from the real torque. 
This is the author's version of an article that has been published in the Proceedings of the

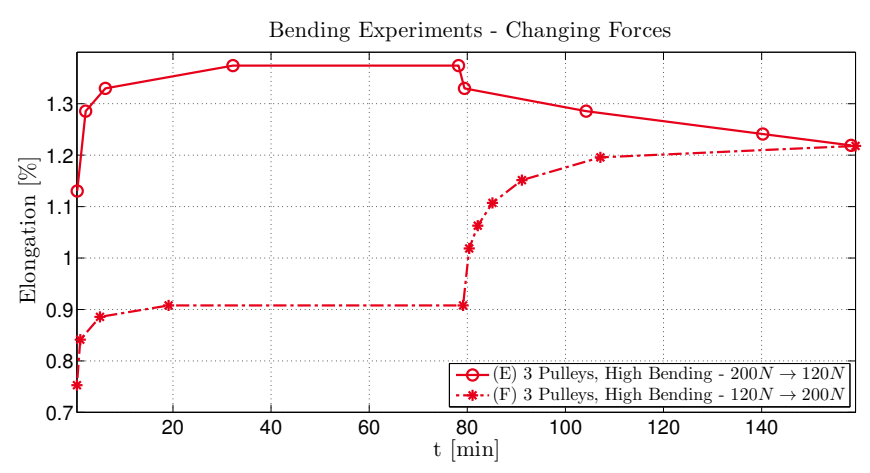

Fig. 5. Elongation results of the bending experiments with changing forces from $200 \mathrm{~N}$ to $120 \mathrm{~N}$ and vice versa. Each force is applied for $80 \mathrm{~min}$.

If a system is equipped with a joint torque sensor, this information can be used to compute the position offset term caused by the tendon elongation using (3) and (4)

$$
\begin{aligned}
\boldsymbol{\theta} & =\boldsymbol{\tau}_{e l} \cdot \boldsymbol{K}^{-1}+\boldsymbol{q} \\
\boldsymbol{\Delta}_{e} & =\hat{\boldsymbol{\theta}}-\boldsymbol{\theta} .
\end{aligned}
$$

In the case of no direct joint torque sensing, a model based estimation can be done based on the motor dynamics (2) or rigid body dynamics (1) like in [20] for joint stiffness estimation. The drive train friction of tendon driven robots is hard to model and identify because of the load dependency and absence of joint torque sensing, thus, an observer-based approach as in [21] is not applicable. So, the elastic joint torque estimation based on the motor dynamics that needs friction information is not regarded here.

Inspired by [18] and [20], the robot's rigid body dynamics and the generalized momentum can be used alternatively. Using (1) and a stable, first order low-pass filter leads to the elastic joint torque observer

$$
\begin{aligned}
\boldsymbol{\tau}_{e l} & =\boldsymbol{K}_{I}\left(\boldsymbol{p}-\int_{0}^{t}\left(\boldsymbol{\tau}_{e x t}+\boldsymbol{\alpha}(\boldsymbol{q}, \dot{\boldsymbol{q}})+\boldsymbol{\tau}_{e l}\right) d t\right) \\
\boldsymbol{\alpha}(\boldsymbol{q}, \dot{\boldsymbol{q}}) & =\boldsymbol{C}(\boldsymbol{q}, \dot{\boldsymbol{q}})^{T} \dot{\boldsymbol{q}}-\boldsymbol{g}(\boldsymbol{q})-\boldsymbol{\tau}_{f, j}
\end{aligned}
$$

with the positive diagonal matrix $\boldsymbol{K}_{I}$ that determines the filter's reactivity and the generalized momentum $\boldsymbol{p}=\boldsymbol{M}(\boldsymbol{q}) \ddot{\boldsymbol{q}}$.

Using the equations (7), (5) and (6), one obtains an estimation of the position offset $\hat{\boldsymbol{\Delta}}_{e}$ that is caused by the tendon elongation. If the external forces are not known the position offset is overestimated. This has to be regarded if the estimation is used for wear analysis.

\section{EXPERIMENTAL EVAluation OF TENDON ELONGATION ESTIMATION}

In order to evaluate the capability of the proposed tendon elongation estimation, experiments in simulation and with a real robot have been performed. The elongation position offset $\Delta_{e}$ is added to the real motor position $\theta$ if the position is larger that $\Delta_{e}$ and zero otherwise, to simulate the tendon elongation. Additionally, the influence of inaccuracies in the spring stiffness coefficient and the dynamics model are regarded. For the robot experiments the ultra lightweight tendon driven series elastic robot arm BioRob ([10]) is used.

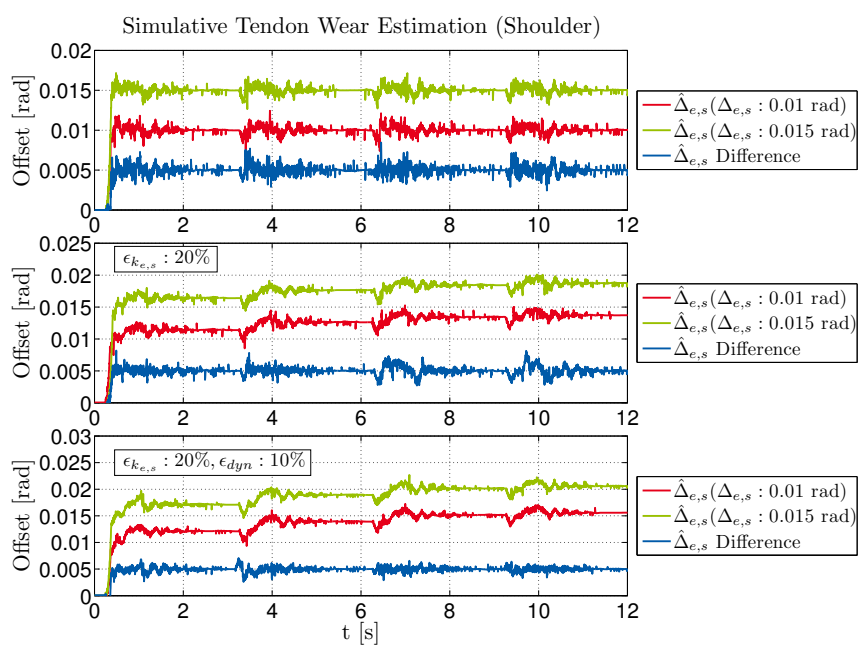

Fig. 6. Tendon wear estimation results in simulation, estimated as motor position offset with different model errors

The elongation position offset estimations are depicted in Fig. 6 showing the results during a motion of the shoulder joint from zero to $90^{\circ}$ in four position steps. First, only a position offset $\Delta_{e, s}$ in the shoulder is present and increased from $0.01 \mathrm{rad}$ to $0.015 \mathrm{rad}$. Fig. 6 (top) shows that the estimation is accurate except at the beginning, which is caused by the implementation of the position offset as described in the previous paragraph.

The offset estimations with errors in the shoulder spring stiffness coefficient $\epsilon_{k_{e, s}}$ of $20 \%$ (overestimation) and with $10 \%$ (underestimation) for each dynamics matrix/vector $\epsilon_{d y n}$ are shown in in Fig. 6 (middle) and (bottom). The absolute position offset value is no longer correct. Nevertheless, the proposed estimation is able to estimate the position offset changes accurately (see $\hat{\Delta}_{e, s}$ Difference).

The same motion as in simulation but for the elbow joint has been performed on the BioRob arm. Since the tendon elongation is not precisely adjustable, it is realized as in simulation by adding the position offset in the elbow $\Delta_{e, e}=0.01 \mathrm{rad}$ to the measured motor position. In the joint torque estimation (7) the unknown friction torques $\tau_{f, j}$ are set to zero. The results in Fig. 7 (top) show similar estimation characteristics as in simulation with model errors (Fig. 6 bottom), but with decreasing tendency. This is caused by an overestimated joint torque (resulting from model inaccuracies), underestimated spring stiffness, or a combination of both in (5). Nevertheless, the estimation is able to detect the absolute degradation (Fig. 7 middle), but with increased noise and oscillation caused by the real sensors and elasticities.

In the second robot experiment, the position offset is introduced by relaxing the rope with the existing rope tensioner by approximately $0.5 \mathrm{~mm}$. The estimation results in Fig. 7 (bottom) show that the position offset does not start from zero, as expected, and after re-tensioning the rope the initial estimation is reached again.

The presented evaluation shows that the proposed estima- 

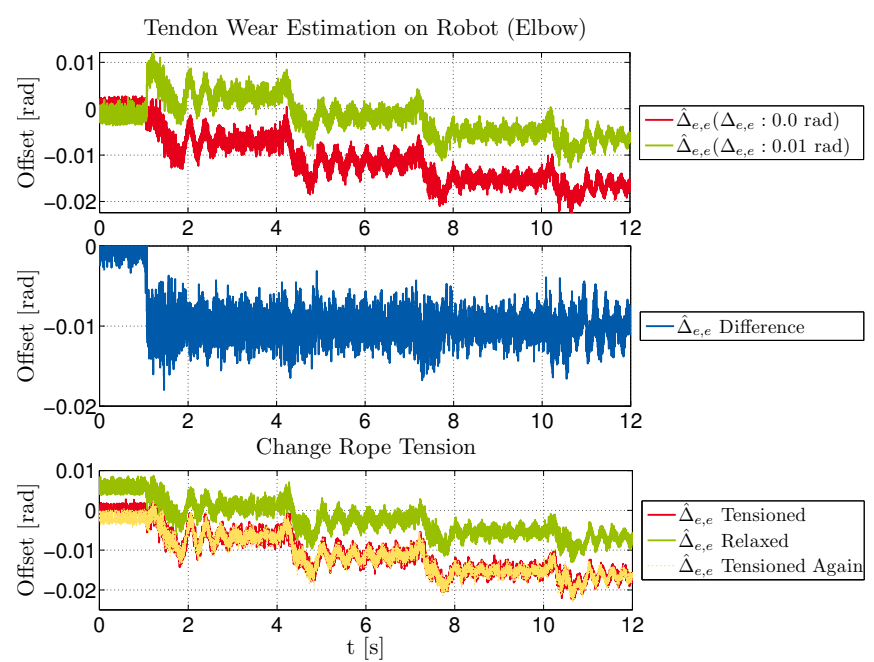

Fig. 7. Tendon wear estimation results on Robot, estimated as motor position offset.

tion mechanism is well suited for long term monitoring of the position offset caused by tendon degradation for quality assurance and to avoid failures.

\section{CONCLUSION}

The selection of ropes for tendon driven actuation systems is a challenging task because of the variety of possible materials and the absence of detailed characteristics of a specific rope. In this work we presented a new comparative evaluation of ropes consisting of the state-of-the-art synthetic fibers in a diameter range from $1.22 \mathrm{~mm}$ to $2.05 \mathrm{~mm}$ from different manufacturers suitable for use in lightweight tendon driven actuation. The results show that the rope elongation does not only dependent on the fundamental characteristic of the used high performance fiber, but also on the braiding and production process. A larger diameter does not necessarily mean a smaller elongation regarding the same rope force.

Further, we investigated the elongation characteristics of a rope, made of the commonly used HMPA fiber readily available on the marked, during bending experiments with constant and changing forces. It was shown in several general arrangements of guiding pulleys, that redirecting the rope does influences the bending characteristics. Especially, stressing a portion of the rope more than once, or a low wrap angle result in a higher elongation. The elongation behavior at different forces suggest that an appropriate pretension of the ropes can reduce the elongation effects.

The knowledge gained from the performed rope analysis strongly supports the design process of tendon driven robots.

In order to detect the tendon elongation for tendon driven joint elastic robot arms, we introduced an observer-based approach. This approach does only need motor and joint position measurements and is also able to detect wear in the presence of model errors for quality assurance, thus, avoid failures and increase reliability. The detection performance has been evaluated in simulation and via robot experiments.

\section{ACKNOWLEDGMENT}

This work has been partially supported by the Hessian State Ministry of Higher Education, Research and the Arts (HMWK) under grant 438/14-28.

The authors would like to thank Mr. Haupt and Mr. Klein from MTI for the good cooperation and Nicola Rupf for preparation of the test bench design data.

\section{REFERENCES}

[1] ISO/TS 15066, Robots and robotic devices - Collaborative robots, Technical Specification ISO/TS 15 066:2016, February 2016.

[2] D. Scholz, C. Maufroy, S. Kurowski, K. Radkhah, O. von Stryk, and A. Seyfarth, "Simulation and experimental evaluation of the contribution of biarticular gastrocnemius structure to joint synchronization in human-inspired three-segmented elastic legs," in 3rd Int. Conf. on Sim., Mod. and Program. for Auton. Robots (SIMPAR), 2012, pp. 251-260.

[3] S. Jacobsen, E. Iversen, D. Knutti, R. Johnson, and K. Biggers, "Design of the utah/m.i.t. dextrous hand," in IEEE Int. Conf. on Robotics and Automation, vol. 3, Apr 1986, pp. 1520-1532.

[4] F. Lotti, P. Tiezzi, G. Vassura, L. Biagiotti, and C. Melchiorri, "Ubh 3: an anthropomorphic hand with simplified endo-skeletal structure and soft continuous fingerpads," in IEEE Int. Conf. on Robotics and Automation, vol. 5, April 2004, pp. 4736-4741 Vol.5.

[5] J. D. Crisman, C. Kanojia, and I. Zeid, "Graspar: a flexible, easily controllable robotic hand," IEEE Robotics Automation Magazine, vol. 3, no. 2, pp. 32-38, Jun 1996.

[6] A. Mazumdar, S. J. Spencer, C. Hobart, J. Dabling, T. Blada, K. Dullea, M. Kuehl, and S. P. Buerger, "Synthetic fiber capstan drives for highly efficient, torque controlled, robotic applications," IEEE Robotics and Autom. Letters, vol. 2, no. 2, pp. 554-561, April 2017.

[7] K. Radkhah, C. Maufroy, M. Maus, D. Scholz, A. Seyfarth, and O. von Stryk, "Concept and design of the biobiped1 robot for human-like walking and running," Int. J. of Humanoid Robotics, vol. 8, no. 3, pp. 439-458, 2011.

[8] Carbon Robotics, https://www.carbon.ai/.

[9] Igus Robolink, http://www.igus.eu/wpck/6076/robolink.

[10] T. Lens, J. Kunz, C. Trommer, A. Karguth, and O. von Stryk, "Biorobarm: A quickly deployable and intrinsically safe, light- weight robot arm for service robotics applications," in 41st Int. Symposium on Robotics (ISR 2010) / 6th German Conf. on Robotics (ROBOTIK 2010), Munich, Germany, Jun 2010, pp. 905-910.

[11] R. Müller, M. Hessinger, H. Schlaak, and P. Pott, "Modelling and characterisation of twisted string actuation for usage in active knee orthoses," IFAC-PapersOnLine, vol. 48, no. 20, pp. 207 - 212, 2015.

[12] S. Rope, "Rope user's manual: Guide to rope selection, handling, usage, and retirement," Samson Rope, Ferndale, WA, USA, Tech. Rep. No. 109101 2.5K 10/2016, 2016.

[13] M. P. Summers and J. W. Hurst, "Rope selection for rope drive transmissions used in robotic manipulation," 2010.

[14] DIN EN ISO 899-1, Determination of creep behaviour - Part 1: Tensile creep, Standard ISO 899-1:2003 + Amd.1:2015, June 2015.

[15] DIN EN ISO 291, Plastics - Standard atmospheres for conditioning and testing, Standard ISO 291:2008, August 2008.

[16] M. W. Spong, "Modeling and control of elastic joint robots," J. Dyn. Sys., Meas. and Contr., vol. 109, no. 4, pp. 310-318, 1987.

[17] J. Kirchhoff and O. von Stryk, "Robust trajectory tracking control for an ultra lightweight tendon driven series elastic robot arm," in IEEE Int. Conf. on Advanced Intelligent Mechatronics (AIM), Banff, Alberta, Canada, July 12-15 2016, pp. 1297-1304.

[18] A. De Luca and R. Mattone, "Actuator failure detection and isolation using generalized momenta," in Proc. IEEE/RSJ Int. Conf. Intelligent Robots and Systems, 2003, pp. 634-639.

[19] A. De Luca, A. Albu-Schäffer, S. Haddadin, and G. Hirzinger, "Collision detection and safe reaction with the DLR-III lightweight manipulator arm," in Proc. IEEE/RSJ Int. Conf. Intelligent Robots and Systems, 2003, pp. 634-639.

[20] F. Flacco and A. D. Luca, "Residual-based stiffness estimation in robots with flexible transmissions," in 2011 IEEE International Conference on Robotics and Automation, May 2011, pp. 5541-5547.

[21] L. Le Tien, A. Albu-Schäffer, A. De Luca, and G. Hirzinger, "Friction observer and compensation for control of robots with joint torque measurement," in Proc. IEEE/RSJ Int. Conf. Intelligent Robots and Systems, Sept 2008, pp. 3789-3795. 